04

\title{
Устойчивые и неустойчивые режимы плазменных диодов при наличии столкновений электронов
}

\author{
(C) S. Pramanik, ${ }^{1}$ В.И. Кузнецов, ${ }^{2}$ N. Chakrabarti ${ }^{3}$ \\ ${ }^{1}$ Department of Applied Mathematics, University of Calcutta, \\ 700009 Kolkata, India \\ ${ }^{2}$ Физико-технический институт им. А.Ф. Иоффре РАН, \\ 194021 Санкт-Петербург, Россия \\ ${ }^{3}$ Saha Institute of Nuclear Physics, \\ 700064 Kolkata, India \\ e-mail: victor.kuznetsov@mail.ioffe.ru
}

Поступило в Редакцию 8 февраля 2019 г.

В окончательной редакции 8 фревраля 2019 г.

Принято к публикации 22 апреля 2019 г.

Термоэмиссионный преобразователь энергии моделируется с помощью диода Пирса, у которого межэлектродный промежуток заполнен однородно распределенными неподвижными ионами. Исследована устойчивость стационарных состояний такого диода при наличии столкновений электронов с фоновым газом. Стационарные решения получены аналитически с помощью метода Лагранжа. С использованием теории возмущений выведено дисперсионное уравнение. Построены как апериодические, так и колебательные решения этого уравнения и изучены их свойства. Определены области, где апериодические моды неустойчивы. Показано, что все колебательные моды устойчивы.

Ключевые слова: плазменный диод, столкновения электронов.

DOI: $10.21883 / J T F .2019 .10 .48169 .46-19$

\section{Введение}

Изучение вакуумного диода с потоком электронов в режиме ограничения тока пространственным зарядом началось с пионерских работ Чайлда и Ленгмюра [1,2]. В дальнейшем их теоретические исследования были обобщены на случай, когда электронный поток имеет конечную величину скорости инжекции [3]. Позднее Пирс изучал диод, в котором присутствует однородный ионный фон [4]. Авторы обнаружили, что существует пороговая величина плотности тока, при превышении которой в системе развивается апериодическая неустойчивость (неустойчивость Бурсиана-Пирса), и в результате ток, проходящий через диод, существенно уменьшается [5]. Этот предел получил название „space-charge-limit" точка (точка SCL) [6].

В последние несколько десятков лет процессы в плазменных диодах активно изучаются в связи с широкой областью их применения в современной технике [7-14]. Это, например, такие устройства как термоэмиссионный преобразователь энергии (ТЭП) [15], газонаполненные диоды для термоядерного синтеза [16], лазерные принтеры [17], генераторы СВЧ излучения [18] и многие другие устройства, в основе работы которых лежит плазменный диод.

Стационарные состояния плазменных диодов удобно представлять точками на параметрической плоскости $\left\{\varepsilon_{0}, \delta\right\}$, где $\varepsilon_{0}$ и $\delta-$ напряженность электрического поля у эмиттера и межэлектродный зазор, соответственно [19-23]. Ветви решений стационарных уравнений диода Бурсиана (диода, в котором пучок электронов движется в отсутствие ионного фона), построенные на этой плоскости, называются семейством Бурсиана. В случае присутствия однородного ионного фона (диод Пирса) стационарным состояниям соответствуют два семейства решений: бурсиановское и небурсиановское. Когда поток электронов является чисто монокинетическим, бурсиановское семейство состоит из двух ветвей: NormalC ветвь (расположенная ниже точки SCL) и C-Overlap ветвь (расположенная выше точки SCL). При этом COverlap ветвь заканчивается в точке 0 , соответствующей моменту, когда скорость электронов в плазме впервые обращается в нуль [20-23]. Устойчивость решений, соответствующих этим ветвям, изучалась в ряде работ с использованием дисперсионного уравнения [20-28].

В [15] было предложено использовать термоэмиссионный преобразователь энергии в качестве генератора переменного тока. В основе работы такого генератора лежит явление развития неустойчивости Бурсиана-Пирса. Однако, как показывают эксперименты, возникновение столкновений электронов на атомах в межэлектродном зазоре может привести к подавлению этой неустойчивости. Поэтому появление рассеяния электронов может стать препятствием для генерации переменного тока с помощью ТЭП.

В [12] продемонстрировано, что успешно изучать процессы в термоэмиссионном преобразователе энергии можно с помощью плазменного диода пирсовского типа. В недавней работе [29] мы изучали стационарные состояния плазмы в таком диоде в предположении, что 
с поверхности эмиттера поступает монокинетический поток электронов, которые при движении в межэлектродном зазоре испытывают столкновения с атомами. В настоящей работе мы изучаем, как столкновения влияют на устойчивость полученных решений: как из бурсиановского, так и из небурсиановского семейств. Будет показано, что присутствие столкновений оказывет сильное влияние на устойчивость стационарных состояний диода.

Настоящая работа построена следующим образом. В разд. 1 мы выводим основные уравнения в переменных Лагранжа. В разд. 2 приводятся стационарные решения задачи. В разд. 3 с использованием метода малых возмущений выводится дисперсионное уравнение. В раздю 4 изучается устойчивость стационарных решений. И, наконец, в „Заключение“ делаются заключительные замечания.

\section{1. Описание модели и вывод основных уравнений}

Рассмативается одномерный диод, в котором два плоских электрода бесконечной протяженности помещены на расстоянии $d$ друг от друга. Между ними приложена разность потенциалов $U$. Ось $z$ направлена перпендикулярно поверхности эмиттера $(z=0)$. Нерелятивистский моноэнергетический поток электронов с концентрацией $n_{b}$ и скоростью $v_{b}$ вылетает с эмиттера перпендикулярно его поверхности. Электроны движутся в самосогласованном электрическом поле под действием силы трения, возникающей благодаря их столкновениям с ионами и другими частицами. Электрическое поле $E$ вычисляется через скалярный потенциал $\varphi$, который зависит только от кординаты $z$. Собственным магнитным полем электронного тока пренебрегаем.

Предполается, что межэлектродный промежуток однородно заполнен бесконечно тяжелыми ионами с концентрацией $n_{i}$, которые считаются неподвижными. Ионный фон учитывается путем введения безразмерного параметра - степени компенсации

$$
\gamma=n_{i} / n_{b}
$$

Теоретически $\gamma$ может принимать любые значения. Поэтому случай полной компенсации $(\gamma=1)$ является только одним из возможных; такое устройство называется диодом Пирса [20]. Следует также упомянуть, что предельному случаю $\gamma=0$, т. е. когда ионы отсутствуют, соответствует диод Бурсиана.

Для описания состояний плазмы в 1-мерном случае используем систему уравнений, состоящую из уравне- ний неразрывности, движения и Пуассона:

$$
\begin{gathered}
\frac{\partial n}{\partial t}+\nabla(n \mathbf{v})=0 \\
\left(\frac{\partial}{\partial t}+\mathbf{v} \cdot \nabla\right) \mathbf{v}=-\frac{e}{m} \mathbf{E}+\mathbf{F}_{D}(v) \\
\nabla \mathbf{E}=-\frac{e}{\epsilon_{0}}\left(n-n_{i}\right) .
\end{gathered}
$$

Здесь $e$ и $m-$ заряд и масса электрона, а $\epsilon_{0}=8.854 \cdot 10^{-12} \mathrm{C}^{2} / \mathrm{N} \cdot \mathrm{m}^{2}$ - диэлектрическая постоянная. Член $\mathbf{F}_{D}(v)$ в уравнении движения представляет собой силу трения, вызванную столкновениями электронов с другими частицами.

Для того чтобы переписать уравнения (2) в безразмерном виде, мы вводим характерную энергию и длину, которые представляют собой соответственно кинетическую энергию электронов на эмиттере $W_{b}$ и пучковую дебаевскую длину $\lambda_{D}[9,20]$ :

$$
\begin{gathered}
\lambda_{D}=\left[\frac{2 \epsilon_{0} W_{b}}{e^{2} n_{b}}\right]^{1 / 2} \approx 0.3238 \cdot 10^{-2} \frac{V_{b}^{3 / 4}}{j_{b}^{1 / 2}}[\mathrm{~cm}], \\
W_{b}=m v_{b}^{2} / 2 .
\end{gathered}
$$

Здесь плотность тока пучка $j_{b}=e n_{b} v_{b}$ и ускоряющее на пряжение $V_{b}=W_{b} / e=m v_{b}^{2} /(2 e)$, формирующее пучок, выражаются в амперах на квадратный сантиметр и в вольтах соответственно. Безразмерные координата, время, скорость, потенциал и напряженность электрического поля имеют следующий вид: $\xi=z / \lambda_{D}, t=t \omega_{b}$, $u=v / \sqrt{2 W_{b} / m}, \eta=e \varphi /\left(2 W_{b}\right), \varepsilon=e E \lambda_{D} /\left(2 W_{b}\right)$; здесь $\omega_{b}=\left[e^{2} n_{b} /\left(m \epsilon_{0}\right)\right]^{1 / 2}$ представляет собой характерную частоту электронного пучка. Безразмерные величины межэлектродного расстояния и разности потенциалов между электродами обозначаюся соответственно $\delta$ и $V$. Мы предполагаем также, что величина силы трения пропорциональна скорости электронов и направлена протовоположно ей. Отметим, что в присутствии ионного фона решения зависят также от параметра $\gamma$.

Теперь в безразмерных переменных уравнение (2) принимает следующий вид:

$$
\begin{gathered}
\frac{\partial n}{\partial t}+\frac{\partial}{\partial \xi}(n u)=0, \\
\left(\frac{\partial}{\partial t}+u \frac{\partial}{\partial \xi}\right) u=-\varepsilon-v u, \\
\frac{\partial \varepsilon}{\partial \xi}=-n+\gamma, \quad \varepsilon=-\frac{\partial \eta}{\partial \xi} .
\end{gathered}
$$

Коэффициент $v=\langle v\rangle / \omega_{b}$ будем называть „частотой столкновений“. Здесь $\langle v\rangle=\langle v\rangle /\langle\lambda\rangle-$ средняя частота столкновений, $\langle\lambda\rangle$ - длина свободного пробега электронов, а $\langle v\rangle$ - характерный разброс по скоростям, возникающий в ходе процесса столкновений. В нашей модели $\langle v\rangle=u_{b}\langle\lambda\rangle / \lambda_{D} \sim u_{b} /\left(a \lambda_{D} n_{b}\right)$, где $a-$ сечение рассеяния электронов. Для получения аналитических 
решений мы будем использовать модель Акимова и Шамеля, которую они применяли при изучении влияния столкновений на стационарные решения диода Бурсиана [30]. Поэтому будем считать $v$ постоянной величиной.

Будем использовать следующие граничные условия: $n(\xi=0, t)=1, u(\xi=0, t)=1, \quad \eta(\xi=0, t)=0$ и $\eta(\xi=\delta, t)=V$.

Систему нестационарных уравнений (4) будем изучать, используя метод Лагранжа [20,21,24-27]. Введем „функцию тока“ $t_{0}(\xi, t)$, которая удовлетворяет следующим условиям: $\partial_{\xi} t_{0} \equiv-n$ и $\partial_{t} t_{0}=n u$ (здесь $\partial_{y}$ обозначает частную производную по $y$ ). Физически $t_{0}$ имеет смысл момента вылета электрона с эмиттеpa, т.е. $\zeta\left(t=t_{0}\right)=0$. Это легко проверить, поскольку $(d / d t) t_{0} \equiv \partial_{t} t_{0}+u \partial_{\xi} t_{0}=0$, и, следовательно, „, $t_{0}{ }^{\text {“ }}$ сохраняется вдоль траектории электрона.

При переходе от переменных Эйлера $(\xi, t)$ к переменным Лагранжа $\left(t_{0}, t\right)$ дифференциальные операторы преобразуются в соответствии с формулами $\partial_{\xi} \equiv-n\left(t_{0}, t\right) \partial_{t_{0}} \quad$ и $\quad \partial_{t} \equiv \partial_{t}+n\left(t_{0}, t\right) u\left(t_{0}, t\right) \partial_{t_{0}}$. Следовательно, уравнения (4) принимают следующий вид:

$$
\begin{array}{cc}
\frac{\partial n}{\partial t}-n^{2} \frac{\partial u}{\partial t_{0}}=0, & \frac{\partial u}{\partial t}=-\varepsilon-v u, \\
\frac{\partial \varepsilon}{\partial t_{0}}=1-\frac{\gamma}{n}, & \frac{\partial \eta}{\partial t_{0}}=\frac{\varepsilon}{n} .
\end{array}
$$

Из 3-го уравнения системы (5) получаем

$$
\varepsilon\left(t, t_{0}\right)=-\left(t-t_{0}\right)+\varepsilon_{0}(t)-\gamma \int_{t}^{t_{0}} d t_{0} / n .
$$

Из 1-го уравнения системы (5) следует, что $\xi^{\prime} \equiv \partial_{t_{0}} \xi=-1 / n\left(t, t_{0}\right)$, так что

$$
\varepsilon\left(t, t_{0}\right)=-\left(t-t_{0}\right)+\varepsilon_{0}(t)+\gamma \xi\left(t, t_{0}\right) .
$$

Используя соотношение $u=\partial_{t} \xi$, из первых двух уравнений системы (5) и формулы (6), получаем следующее уравнение для положения электрона:

$$
\frac{\partial^{2} \xi}{\partial t^{2}}+v \frac{\partial \xi}{\partial t}+\gamma \xi=\left(t-t_{0}\right)-\varepsilon_{0}(t) .
$$

\section{2. Стационарные решения}

В стационарном случае напряженность электрического поля у эмиттера $\varepsilon_{0}$ не зависит от времени, а другие величины зависят только от разности $\tau=t-t_{0}$. Следовательно:

$$
\varepsilon(\tau)=-\tau+\varepsilon_{0}+\gamma \zeta(\tau) .
$$

Операторы частных производных преобразуются следующим образом: $\partial_{t} \rightarrow \partial_{\tau}$ и $\partial_{t_{0}} \rightarrow-\partial_{\tau}$. Тогда уравнение (7) принимает вид

$$
\frac{d^{2} \zeta}{d \tau^{2}}+\nu \frac{d \xi}{d \tau}+\gamma \xi=\tau-\varepsilon_{0}
$$
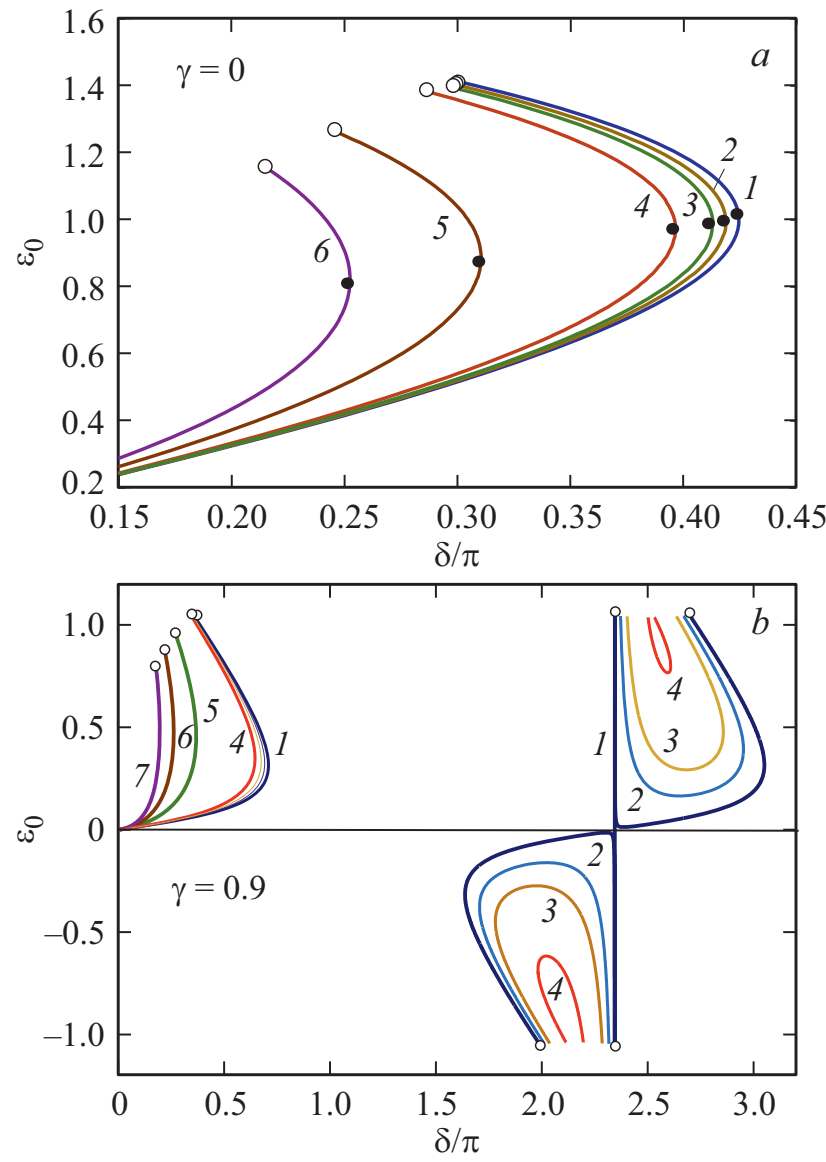

Рис. 1. Зависимость напряженности электрического поля на эмиттере $\varepsilon_{0}$ от межэлектродного зазора $\delta$ для стационарных решений диода с $V=0$ для двух величин $\gamma$ и ряда значений $v: a-\gamma=0 ; v=0$ (кривая 1), 0.02 (2), 0.04 (3), 0.1 (4), $0.5(5), 0.9(6) ; b-\gamma=0.9 ; v=0$ (кривая 1), 0.01 (2), 0.02 (3) $0.04(4), 0.2(5), 0.5(6), 1$ (7). Каждая кривая закончена в точке 0 .

Решая уравнение (9) с начальными условиями $\zeta(\tau=0)=0, d \xi / d \tau(\tau=0)=1$, для случая $v<2 \sqrt{\gamma}$ получаем стационарное решение

$$
\begin{aligned}
& \xi(\tau)=\frac{1}{\gamma} \tau-\frac{1}{\gamma^{2}}\left(\gamma \varepsilon_{0}+v\right)+\frac{1}{\gamma^{2}} \exp \left(-\frac{v}{2} \tau\right) \\
& \times\left\{\left(\gamma \varepsilon_{0}+v\right) \cos (\beta \tau)+\frac{1}{\beta}\left[\frac{\nu}{2}\left(\gamma \varepsilon_{0}+v\right)-\gamma(1-\gamma)\right] \sin (\beta \tau)\right\} .
\end{aligned}
$$

С другой стороны, для случая $v>2 \sqrt{\gamma}$ функция $\xi(\tau)$ принимает вид

$$
\begin{aligned}
& \zeta(\tau)=\frac{1}{\gamma} \tau-\frac{1}{\gamma^{2}}\left(\gamma \varepsilon_{0}+v\right)-\frac{1}{\alpha \gamma^{2}} \\
& \times\left[\frac{1}{2}(\nu-\alpha)\left(\gamma \varepsilon_{0}+v\right)-\gamma(1-\gamma)\right] \exp \left(-\frac{v+\alpha}{2} \tau\right) \\
& +\frac{1}{\alpha \gamma^{2}}\left[\frac{1}{2}(\nu+\alpha)\left(\gamma \varepsilon_{0}+v\right)-\gamma(1-\gamma)\right] \exp \left(-\frac{v-\alpha}{2} \tau\right) .
\end{aligned}
$$


Здесь введены две эффективные „частоты“:

$$
\beta=\sqrt{\gamma-v^{2} / 4}, \quad \alpha=\sqrt{v^{2}-4 \gamma} .
$$

И, наконец, из соотношения $d \eta / d \tau=-u \varepsilon$ с использованием (8) для потенциала получаем

$$
\eta(\tau)=-\frac{1}{2} \gamma \xi^{2}(\tau)+\left(\tau-\varepsilon_{0}\right) \xi(\tau)-\int_{0}^{\tau} \xi(t) d t .
$$

Для заданных величин $\gamma, v$ и $\varepsilon_{0}$ мы можем рассчитать распределения скорости и концентрации электронов, потенциала и электрического поля в межэлектродном промежутке, увеличивая постепенно величину $\tau$. Обозначая время пролета электронов между электродами через $T$, получаем $\tau=T, \xi=\delta$ и

$$
V=\eta(\tau=T)=-\frac{1}{2} \gamma \delta^{2}+\left(T-\varepsilon_{0}\right) \delta-\int_{0}^{T} \xi\left(t ; \varepsilon_{0}\right) d t
$$

На рис. 1 на плоскости $\left\{\varepsilon_{0}, \delta\right\}$ в параметрическом виде представлены стационарные решения диода Бурсиана $(\gamma=0)$ и обобщенного диода Пирса $(\gamma=0.9)$ для ряда значений $v$. Набор таких кривых дает семейство решений $[14,20-23]$, Как видно из рис. $1, a$, у диода Бурсиана имеется только один тип решений (для $\varepsilon_{0}>0$ и $\delta<1.5)$, а кривые, представляющие их, называются бурсиановскими ветвями [14,23]. С другой стороны, из рис. $1, b$, где представлены решения обобщенного диода Пирса $(\gamma \neq 0)$, видно наличие двух типов решений: бурсиановское и небурсиановское семейства [20,29]. Бурсиановские ветви обобщенного диода Пирса лежат в левой части рис. $1, b$ (для $\delta \leq \pi$ и $\varepsilon_{0}>0$ ), а небурсиановские ветви расположены в области $1<\delta / \pi<3$. Мы можем видеть также, что в отличие от диода Бурсиана стационарные решения диода Пирса существуют и для отрицательных значений $\varepsilon_{0}$. Видно также, что небурсиановские ветви существуют только при малых значениях частоты столкновений, и исчезают, когда $v$ увеличивается.

На правой границе каждой бурсиановской кривой существует точка (точка SCL), где производная $d \delta / d \varepsilon_{0}=0$ (на рис. 1 она помечена жирной точкой). Она относится к стационарному решению, которому соответствует максимальный ток, проходящий через диод $\left(J_{\mathrm{SCL}} \sim \delta_{\mathrm{SCL}}^{2}\right)$. C ростом $v$ величина максимального тока уменьшается. Каждая бурсиановская кривая заканчивается в точке нуль. Эта точка соответствует состоянию диода, у которого в некоторой точке внутри межэлектродного зазора скорость электронов обращается в нуль из-за возникшего виртуального катода. В этом случае вследствие моноэнергетичности пучка все электроны отражаются от виртуального катода и возвращаются на эмиттер. Участок бурсиановской ветви, лежащий между точками SCL и $0\left(\varepsilon_{0, \mathrm{SCL}}<\varepsilon_{0} \leq \varepsilon_{0,0}\right)$, называется C-Overlap ветвью, а лежащий ниже точки $\mathrm{SCL}\left(\varepsilon_{0} \leq \varepsilon_{0, \mathrm{SCL}}\right)-$ Normal $\mathrm{C}$

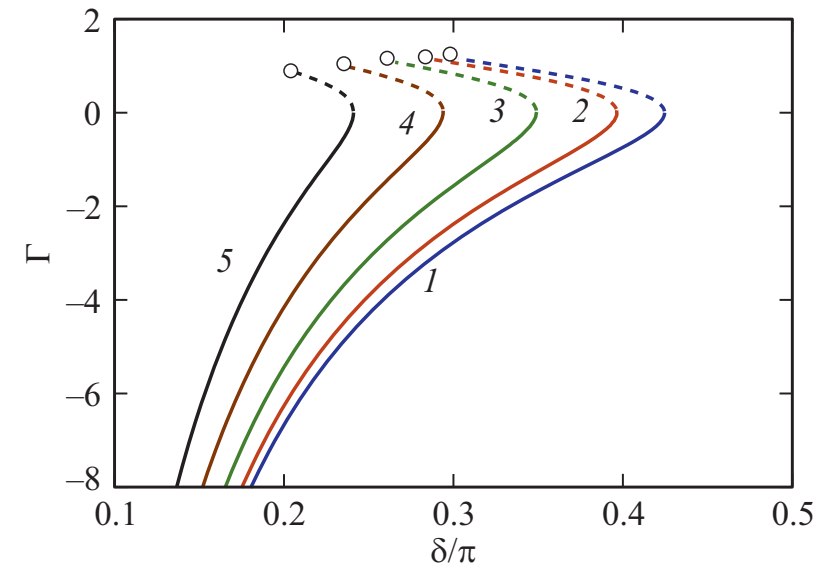

Рис. 2. Апериодические ветви $(\Omega=0)$ диода Бурсиана с $V=0$ для ряда величин частоты столкновений $v: v=0$ (кривая 1), 0.1 (2), 0.3 (3), 0.6 (4), 1.0 (5). Сплошные кривые соответствуют Normal C ветви, штриховые - C-Overlap ветви.

ветвью. Формулы для расчета координат точек SCL и 0 приведены в работе [29].

Для того чтобы проанализировать устойчивость стационарных решений, в разд. 3 выводится дисперсионное уравнение.

\section{3. Вывод дисперсионного уравнения}

Считаем, что стационарные решения диода описываются уравнениями (10) или (11). Наложим на эти решения малое возмущение, пропорциональное $\exp (\sigma t)$, таким же образом, как это делалось в работах $[21,27,28]$. Тогда к электрическому полю на эмиттере и другим стационарным величинам добавится возмущение:

$$
\begin{gathered}
\tilde{\varepsilon}_{0}(\tau, t)=\varepsilon_{0}+\varepsilon_{1}(\tau) \exp (\sigma t), \\
\tilde{\zeta}(\tau, t)=\xi(\tau)+\xi_{1}(\tau) \exp (\sigma t), \\
\tilde{T}(\tau, t)=T+T_{1}(\tau) \exp (\sigma t) .
\end{gathered}
$$

Здесь $\varepsilon_{0}, \xi(\tau)$ и $T$ являются членами нулевого порядка (они определяются уравнениями (10) или (11)), а $\varepsilon_{1}$, $\xi_{1}$ и $T_{1}$ являются величинами 1-го порядка малости по возмущению. Поэтому с точностью до членов 1го порядка напряженность электрического поля можно записать в виде $\varepsilon=\varepsilon^{(0)}+\varepsilon^{(1)}$, где $\varepsilon^{(0)}=\varepsilon_{0}-\tau+\gamma \zeta$, $\underset{\tilde{\xi}}{\mathrm{a}} \varepsilon^{(1)}=\left(\varepsilon_{1}+\gamma \xi_{1}\right) \exp (\sigma t) \quad$ (см. уравнение $\left.(8)\right)$. Тогда $\tilde{\zeta}(\tau, t)$ должно удовлетворять уравнению

$$
\frac{\partial^{2} \tilde{\zeta}}{\partial \tau^{2}}+v \frac{\partial \tilde{\xi}}{\partial \tau}=-\varepsilon(\tau, t)
$$

В 1-м порядке из (16) получаем

$$
\frac{d^{2} \xi_{1}}{d \tau^{2}}+(2 \sigma+v) \frac{d \xi_{1}}{d \tau}+\left(\sigma^{2}+\gamma+v \sigma\right) \xi_{1}=-\varepsilon_{1} .
$$



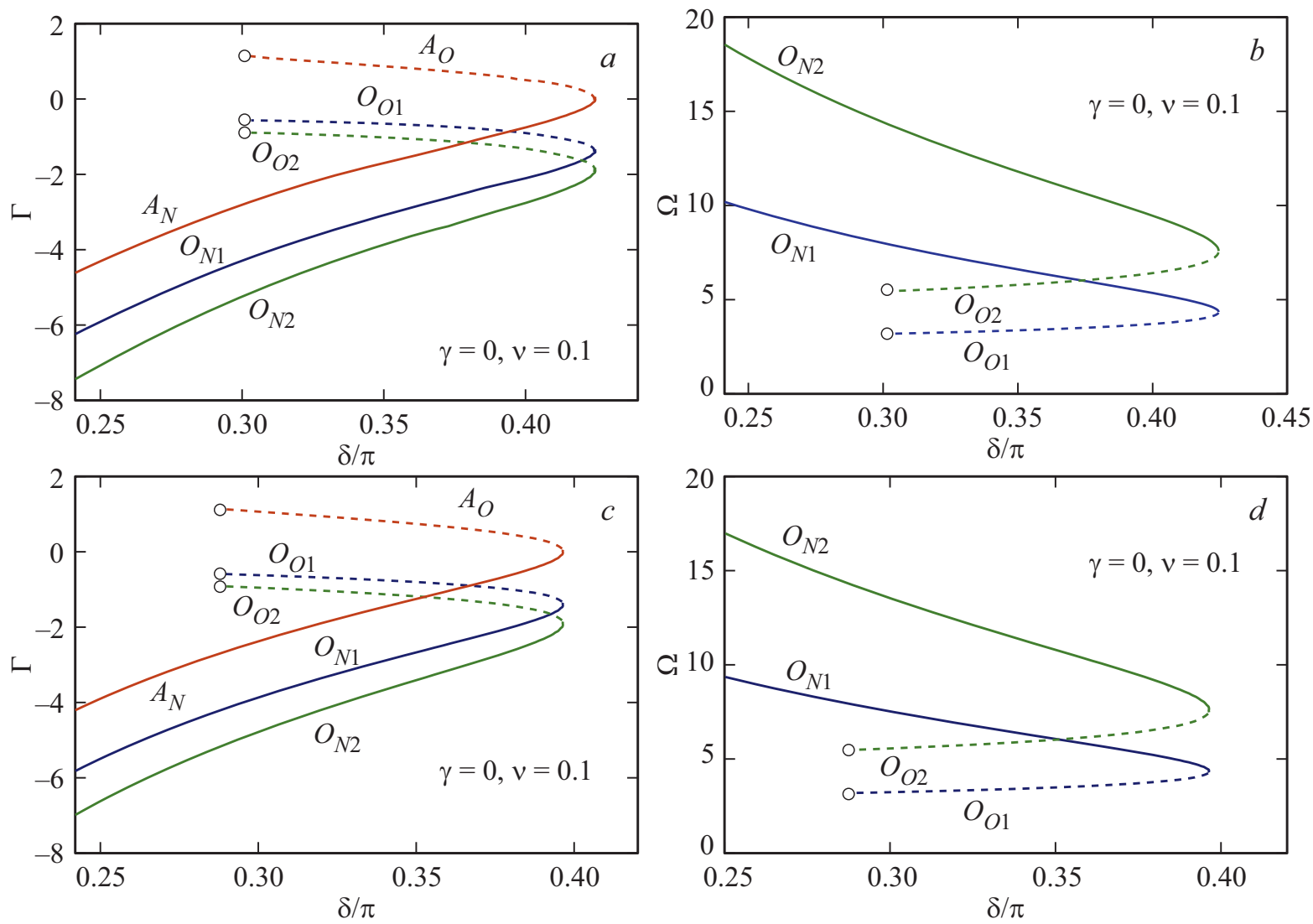

Рис. 3. Зависимости от $\delta / \pi$ вещественной $(\Gamma)(a, c)$, и мнимой $(\Omega)(b, d)$ части корней дисперсионного уравнения для диода Бурсиана с $V=0 ;(a),(b): v=0,(c),(d): v=0.1$. Сплошные кривые соответствуют Normal C ветви, штриховые - C-Overlap ветви. Индекс $A$ обозначает апериодическую моду $(\Omega=0), O-$ колебательную $(\Omega \neq 0)$; $N$ относится к Normal C ветви, а $O-$ к C-Overlap ветви; 1 и 2 обозначают первые две колебательные моды.

Обозначая $\varkappa=\sigma+v / 2$ и используя начальные условия $\xi_{1}(\tau=0)=0$ и $\partial_{\tau} \xi_{1}(\tau=0)=0$, для $\xi_{1}$ получим следующее решение:

$$
\begin{gathered}
\xi_{1}(\tau)=\frac{\varepsilon_{1}}{\gamma+v \sigma+\sigma^{2}}\left[\exp (-\varkappa \tau)\left(\cos \beta \tau+\frac{\varkappa}{\beta} \sin \beta \tau\right)-1\right] \\
\text { (для } v<2 \sqrt{\gamma})
\end{gathered}
$$

или

$$
\begin{gathered}
\xi_{1}(\tau)=\frac{\varepsilon_{1}}{\gamma+\nu \sigma+\sigma^{2}}\left\{\frac { 1 } { \alpha } \operatorname { e x p } ( - \varkappa \tau ) \left[\left(\varkappa+\frac{\alpha}{2}\right)\right.\right. \\
\left.\left.\times \exp \left(\frac{\alpha}{2} \tau\right)-\left(\varkappa-\frac{\alpha}{2}\right) \exp \left(-\frac{\alpha}{2}-\tau\right)\right]-1\right\} \\
\text { (для } v<2 \sqrt{\gamma}) .
\end{gathered}
$$

Когда электрическое поле на эмиттере возмущается, траектория электронов будет отличаться от стационарной, и, следовательно, изменится время пролета электронов между электродами (скажем, $\tilde{T}=$ $\left.=T+T_{1} \exp (\sigma t)\right)$. В 1-м порядке из условия $\tilde{\zeta}(\tilde{T})=\delta$ получим

$$
T_{1}=-\frac{\xi_{1}(T)}{u(T)}
$$

Теперь для получения дисперсионного уравнения воспользуемся граничным условием для разности потенциалов между электродами. Если она равна $V$, то получаем соотношение

$$
\begin{aligned}
V= & -\int_{0}^{\delta} \varepsilon(\zeta) d \xi=-\int_{0}^{\tilde{T}} \varepsilon(\tau) u(\tau) d \tau \\
& =-\int_{0}^{\tilde{T}}\left[\varepsilon^{(0)}+\varepsilon^{(1)}\right] \partial_{\tau}\left[\zeta^{(0)}+\xi^{(1)}\right] d \tau .
\end{aligned}
$$

Используя соотношение (20), из (21) получаем следующую связь между величинами 1-го порядка

$$
\delta \varepsilon_{1}+\int_{0}^{T} \xi_{1} d \tau=0 .
$$

После подстановки выражения для $\xi_{1}$ из (18) в (22) для случая $v<2 \sqrt{\gamma}$ получаем дисперсионное уравнение в виде

$$
\begin{aligned}
& F(\sigma ; \delta, T)=-\exp (-\varkappa T)\left[2 \varkappa \cos \beta T+\frac{\varkappa^{2}-\beta^{2}}{\beta} \sin \beta T\right] \\
& +\left(\gamma+v \sigma+\sigma^{2}\right)^{2} \delta-\left(\gamma+v \sigma+\sigma^{2}\right) T+2 \varkappa=0
\end{aligned}
$$



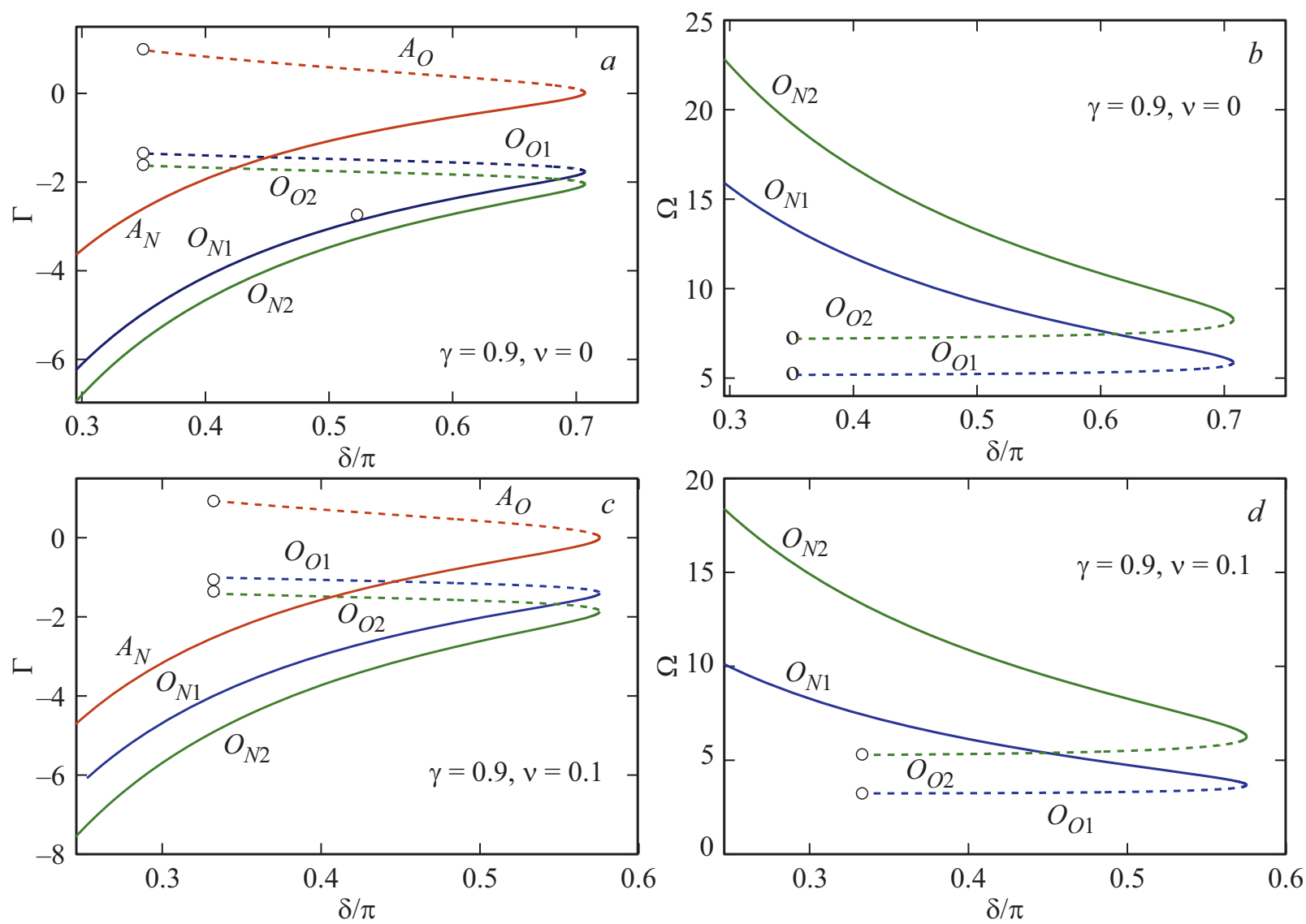

Рис. 4. Зависимости от $\delta / \pi$ вещественной $(a, c)$ и мнимой части $(b, d)$ корней дисперсионного уравнения, соответствующие бурсиановским ветвям обобщенного диода Пирса с $\gamma=0.9$ и $V=0 ;(a, b): v=0,(c, d): v=0.1$. Все индексы имеют тот же смысл, что и на рис. 3.

Аналогично, используя (19) для $\xi_{1}$, получаем дисперсионное уравнение для случая $v>2 \sqrt{\gamma}$

$$
\begin{aligned}
& F(\sigma ; \delta, T)=-\frac{1}{\alpha} \exp (-\varkappa T)\left[\left(\varkappa+\frac{\alpha}{2}\right)^{2} \exp (\alpha T / 2)\right. \\
& \left.-\left(\varkappa-\frac{\alpha}{2}\right)^{2} \exp (-\alpha T / 2)\right]+\left(\gamma+v \sigma+\sigma^{2}\right)^{2} \delta \\
& -\left(\gamma+v \sigma+\sigma^{2}\right) T+2 \varkappa=0 .
\end{aligned}
$$

Следует отметить, что общая форма дисперсионного уравнения (22) является одной и той же для плазменных диодов с пучком электронов всех типов. Это соотношение не зависит от того, присутствует ли ионный фон, поперечное магнитное поле или имеет место рассеяние электронов (в рамках рассматриваемой нами модели). В разных случаях будут только разными выражения для $\xi_{1}[20,21,28]$.

\section{4. Анализ устойчивости решений}

Исследуем решения дисперсионного уравнения (23) (или (24)). Положим $\sigma(\delta ; \gamma, V)=\Gamma(\delta ; \gamma, V)+i \Omega(\delta ; \gamma, V)$.
Вообще говоря, при заданных значениях параметров $\gamma$, $v, V$ и $\delta$ дисперсионное уравнение имеет счетное число собственных мод. Так что уравнение (23) (или (24)) имеет большое число корней. Однако, главной собственной модой (той, которая имеет наибольший инкремент в случае неустойчивого решения или наименьший декремент в случае устойчивого решения) оказывается апериодическая мода. Когда величины параметров $\gamma$ и $V$ заданы, будем называть кривые $\Gamma(\delta ; \gamma, V)$ или $\Omega(\delta ; \gamma, V)$ ветвями неустойчивости.

Сначала рассмотрим апериодические ветви неустойчивости (ветви $A)$. Они определяются корнями уравнения (23) или (24) при вещественной величине $\sigma$ $(\Omega=0 ; \sigma=\Gamma)$. Для случая $v<2 \sqrt{\gamma}$ соответствующее дисперсионное уравнение имеет вид

$$
\begin{aligned}
& F(\Gamma ; \delta, T)=-\exp [-(\Gamma+v / 2) T] \\
& \times\left[2(\Gamma+v / 2) \cos \beta T+\frac{(\Gamma+v / 2)^{2}-\beta^{2}}{\beta} \sin \beta T\right] \\
& +\left(\gamma+v \Gamma+\Gamma^{2}\right)^{2} \delta-\left(\gamma+v \Gamma+\Gamma^{2}\right) T+2 \Gamma+v=0,
\end{aligned}
$$



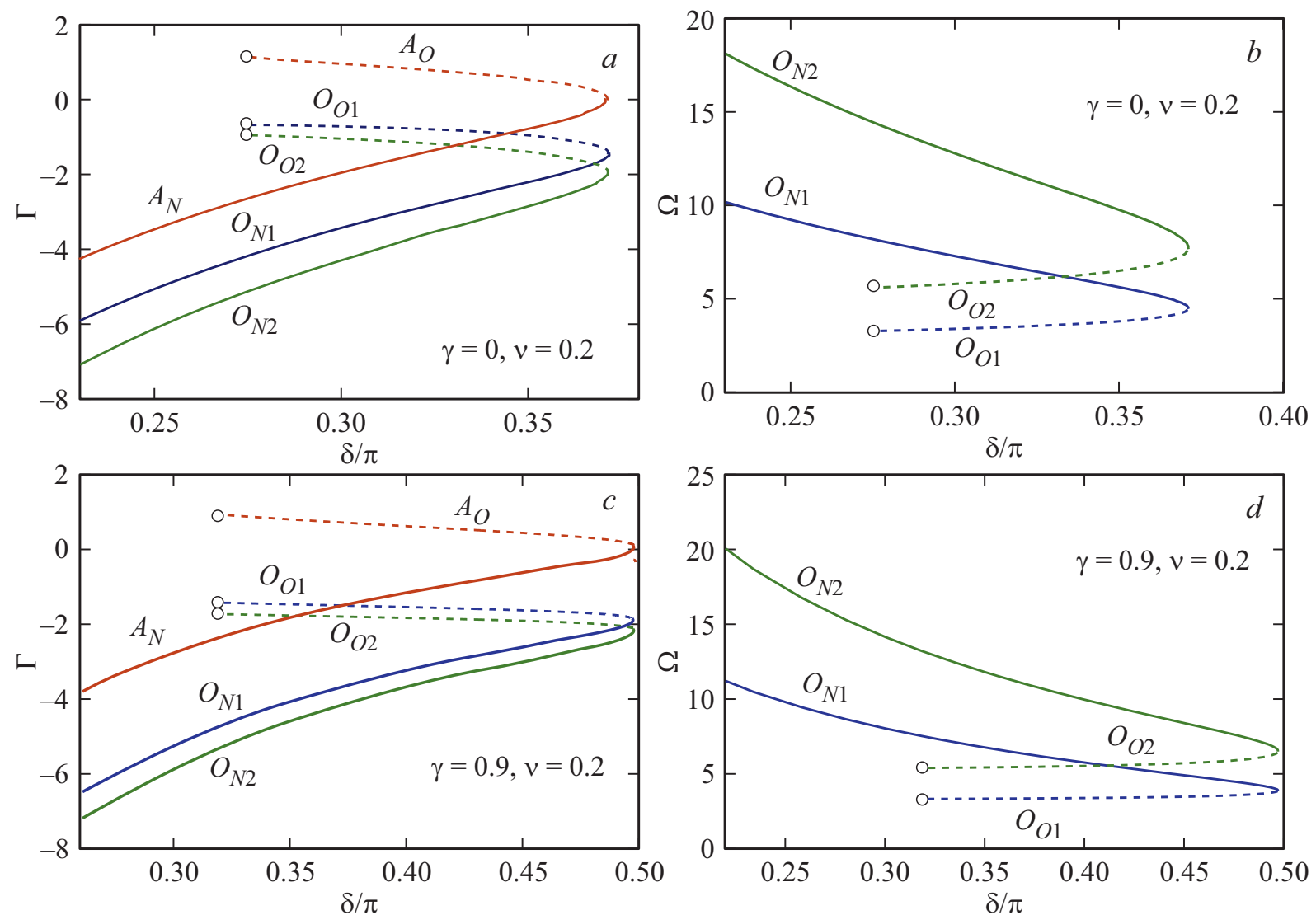

Рис. 5. Зависимости от $\delta / \pi$ вещественной $(a, c)$ и мнимой $(b, d)$ части корней дисперсионного уравнения, соответствующие бурсиановским ветвям диода Бурсиана и обобщенного диода Пирса с $v=0.2$ и $V=0 ;(a, b): \gamma=0,(c, d): \gamma=0.9$. Все индексы имеют тот же смысл, что и на рис. 3.

а для случая $v>2 \sqrt{\gamma}-$

$$
\begin{aligned}
& -\frac{1}{\alpha} \exp \left[-\left(\frac{v}{2}+\Gamma\right) T\right]-\left[\left(\frac{v+\alpha}{2}+\Gamma\right)^{2}\right. \\
& \left.\times \exp \left(\frac{\alpha}{2} T\right)-\left(\frac{v-\alpha}{2}+\Gamma\right)^{2} \exp -\left(-\frac{\alpha}{2} T\right)\right] \\
& +\left(\gamma+v \Gamma+\Gamma^{2}\right)^{2} \delta-\left(\gamma+v \Gamma+\Gamma^{2}\right) T+v+2 \Gamma=0 .
\end{aligned}
$$

Подставляя $\Gamma=0$ и выражение для $\delta$ в уравнение $(25)$, для случая $v<2 \sqrt{\gamma}$ получим

$$
\varepsilon_{0} \cos (\beta T)+\frac{1}{\beta}\left(\frac{\nu}{2} \varepsilon_{0}+\gamma\right) \sin (\beta T)=\varepsilon_{0} \exp \left(\frac{\nu}{2} T\right) .
$$

Из уравнения (26) для случая $v>2 \sqrt{\gamma}$ получим

$$
\begin{aligned}
& \frac{\varepsilon_{0}}{\gamma}+\frac{1}{\alpha \gamma}\left\{\left[\frac{v-\alpha}{2} \varepsilon_{0}+\gamma\right] \exp \left(-\frac{v+\alpha}{2} T\right)\right. \\
& \left.-\left[\frac{\nu+\alpha}{2} \varepsilon_{0}+\gamma\right] \exp \left(-\frac{v-\alpha}{2} T\right)\right\}=0,
\end{aligned}
$$

Если сравнить эти формулы с аналогичными соотношениями для координат точек SCL, полученными в работе [29] (см. формулы (25) и (26)), то можно увидеть, что инкремент апериодической ветви обращается в нуль как раз в точках SCL.

Сначала рассмотрим случай диода Бурсиана $(\gamma=0)$, где существует только один тип решений в области $0<\delta / \pi<1$ на $\left(\varepsilon_{0}, \delta\right)$-диаграмме - бурсиановские ветви. На рис. 2 показаны апериодические ветви такого диода для ряда величин $v$. Каждая ветвь пересекает ось $\Gamma=0$ в точке SCL. Верхняя часть этой ветви заканчивается в точке 0 (где $\varepsilon_{0}=\varepsilon_{0,0}$ ), которая помечена кружком. Очевидно, что стационарные решения, соответствующие верхней части ветви $\left(\varepsilon_{0, \mathrm{SCL}}<\varepsilon_{0} \leq \varepsilon_{0,0}\right)$, или C-overlap ветви, являются неустойчивыми, поскольку имеют положительный инкремент. В то же время, нижняя часть ветви $\left(0<\varepsilon_{0} \leq \varepsilon_{0, \mathrm{SCL}}\right)$, или Normal C ветвь, является устойчивой относительно малых апериодических возмущений (у нее инкремент отрицателен).

Теперь рассмотрим колебательные возмущения $(\Omega \neq 0)$. На рис. 3 наряду с апериодической модой (обозначенной символом $A$ ) показаны две колебательных моды (обозначенные символом $O$ ) для случаев $v=0$ (рис. $3, a$ и $b$ ) и $v=0.1$ (рис. $3 c$ и $d$ ). Индексы $N$ и $O$ относятся к Normal C ветви (сплошная кривая) и C-overlap ветви (штриховая кривая) соответственно. Видно, что 

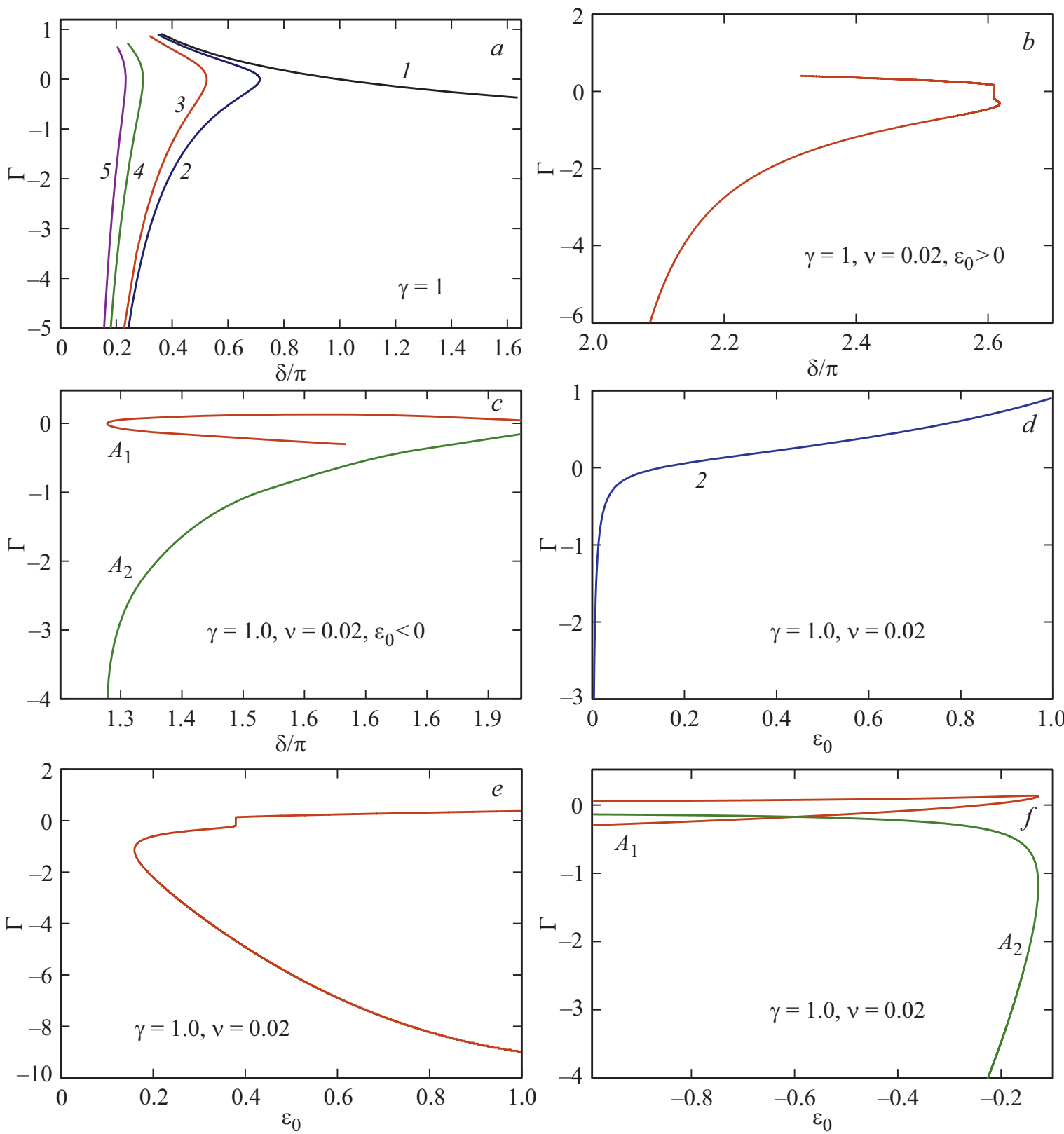

Рис. 6. Диод Пирса с $\gamma=1$ и $V=0: a-$ зависимость инкремента апериодической моды от $\delta / \pi$ для бурсиановской ветви и ряда значений частоты столкновений: $v=0$ (кривая 1), $0.02(2), 0.06(3), 0.8(4), 1.2(5) . d-$ зависимость инкремента апериодической моды от $\varepsilon_{0}$ для бурсиановской ветви при $v=0.02$. Зависимость инкремента апериодической моды $(b)$ от $\delta / \pi$ и $(e)$ от $\varepsilon_{0}$ для небурсиановской ветви с $\varepsilon_{0}>0$ при $v=0.02$. $c$ и $f$ - такие же зависимости, но только для $\varepsilon_{0}<0$. Нижние цифры 1 и 2 соответствуют первым двум модам.

инкременты у колебательных мод отрицательны, т. е. соответствующие решения являются устойчивыми. Таким образом, можно утверждать, что Normal C ветвь является устойчивой относительно любых малых возмущений.

Далее рассмотрим обобщенный диода Пирса. Его стационарные решения располагаются и на бурсиановских, и на небурсиановских семействах решений. Сперва проанализируем бурсиановские ветви. На рис. 4 показаны апериодическая мода $(\sigma=\Gamma)$ и две колебательных моды $(\sigma=\Gamma+i \Omega)$ для $\gamma=0.9$ и двух величин параметра $\nu$ : рис. $4 a$ и $b$ соответствуют $v=0$, а рис. $4 c$ и $d-$ $v=0.1$. И в этом случае мы видим, что Normal C ветвь (сплошная кривая) устойчива (имеет отрицательный инкремент), а C-overlap ветвь (штриховая кривая) - неустойчива относительно малых возмущений (см. красную кривую $A$ на рис. 4, $a$ ), а построенные колебательные ветви - устойчивы. Апериодические и колебательные ветви для $v=0.2$ представлены на рис. $5, a$ и ( $b$ для $\gamma=0$, а для на рис. $5 c$ и $d-$ для $\gamma=0.9$.

На рис. 6, $а$ показаны апериодические дисперсионные кривые для бурсиановских ветвей решений для диода с 

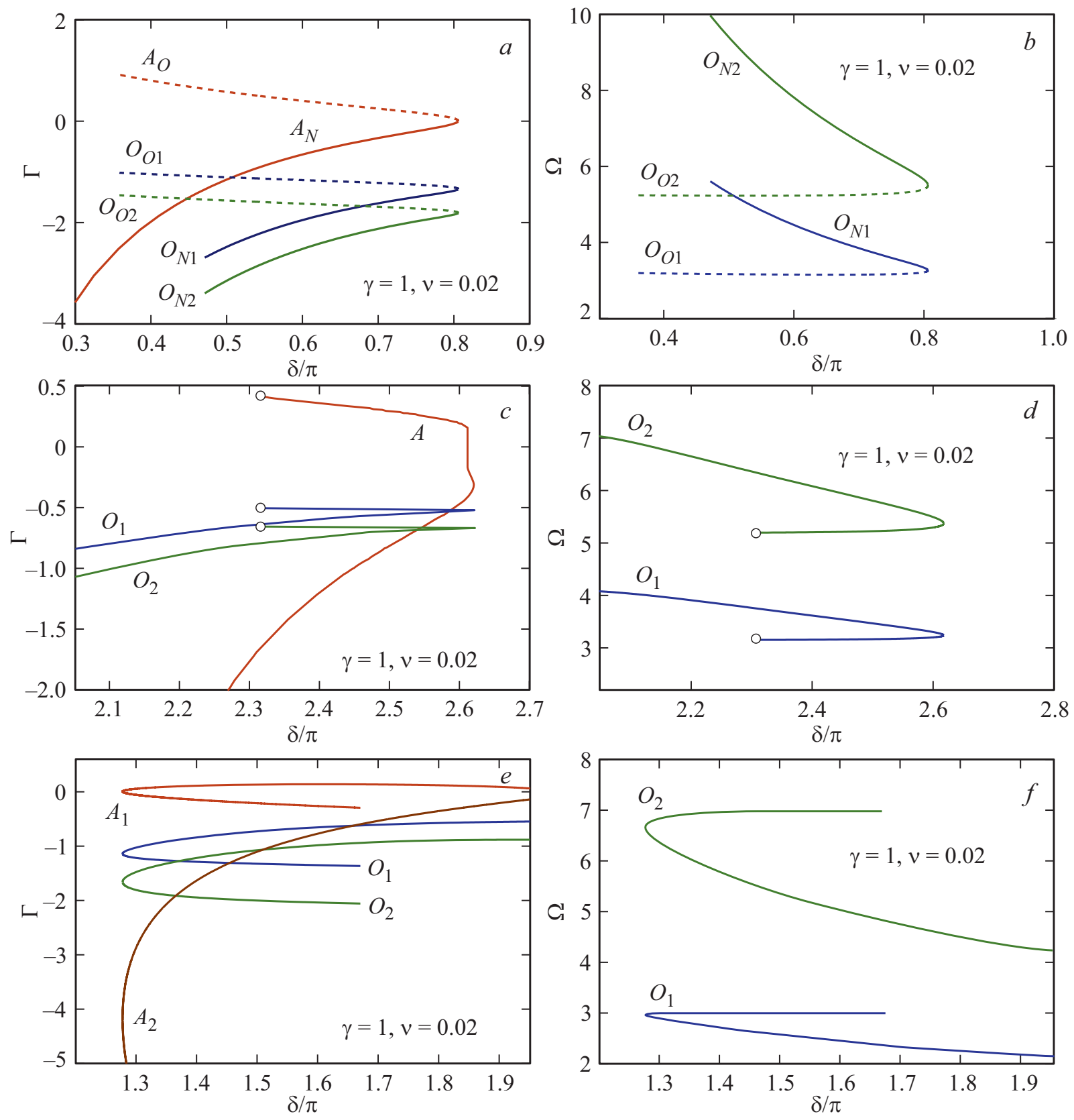

Рис. 7. Дисперсионные ветви диода Пирса, соответствующие $(a)$ и $(b)$ бурсиановскому семейству, небурсиановскому семейству с $\varepsilon_{0}>0(c)$ и $(d)$, небурсиановскому семейству с $\varepsilon_{0}<0(e)$ и $(f)$. Все индексы имеют тот же смысл, что и на рис. 3 и 6.

$\gamma=1$ и ряда значений $v$, а на рис. $6, b-f$ - для небурсиановских ветвей для $\gamma=1$ и $v=0.02$. На рис. $6, b$ и $e$ представлены моды для решений с $\varepsilon_{0}>0$, а на рис. $6, c$ и $f-$ для решений с $\varepsilon_{0}<0$. Символы $A_{1}$ и $A_{2}$ на рис. $6, c$ и $f$ относятся к 1-й и 2-й апериодическим модам соответственно.

На рис. 7 показаны колебательные дисперсионнве кривые как для бурсиановской, так и для небурсиановской ветвей диода с $\gamma=1$ и $v=0.02$. Рис.7, $a$ и $b$ соответствуют бурсиановскому семейству. Колебательные моды небурсиановских семейств представлены двумя группами: рис. 7, $c$ и $d$ соответствуют решениям с $\varepsilon_{0}>0$, а рис. 7, $e$ и $f-\mathrm{c} \varepsilon_{0}<0$. У всех этих кривых участки, где зачения Г положительны, соответствуют неустойчивым решениям, а где они отрицательны - устойчивым. Колебательные моды небурсиановских ветвей всегда устойчивы. Однако часть апериодических мод у этих решений оказывается неустойчивой.

\section{Заключение}

В представленной работе мы изучили устойчивость стационарных решений обобщенного диода Пирса при наличии рассеяния электронов на атомах или других 
частицах. Эти решения получены с помощью метода Лагранжа. Все решения могут быть отнесены либо к бурсиановскому, либо к небурсиановскому семействам в представлении на плоскости $\left\{\varepsilon_{0}, \delta\right\}$. Показано, что состояния, соответствующие небурсиановскому семейству, могут существовать только при сравнительно небольшой частоте столкновений. Для изучения устойчивости найденных решений мы вывели дисперсионное уравнение с помощью теории возмущений. Установлено, что бурсиановские решения, принадлежащие Normal С ветви $\left(\varepsilon_{0}<\varepsilon_{0, \mathrm{SCL}}\right)$, всегда устойчивы относительно малых возмущений, в то время как решения, принадлежащие C-Overlap ветви $\left(\varepsilon_{0}>\varepsilon_{0, \mathrm{SCL}}\right)$, неустойчивы. Когда величина частоты столкновений постепенно начинает увеличиваться, ширина неустойчивой C-Overlap ветви уменьшается. Установлено, что колебательные собственные моды всегда имеют отрацительные инкременты. Для небурсиановских решений найдены также области, где апериодические моды устойчивы и неустойчивы. Показано, что колебательные моды небурсиановских решений всегда устойчивы.

Из экспериментов известно, что при высоких температурах эмиттера максимальная удельная мощность термоэмиссионного преобразователя энергии достигается при давлениях цезия, соответствующих области перехода из бесстолкновительного в столкновительный для электронов режим [31]. Проведенное нами исследование влияния столкновений электронов на стационарные характеристики плазменного диода даст возможность для проведения оптимизации характеристик ТЭП.

С другой стороны, ТЭП может работать в качестве генератора переменного тока только в том случае, когда в плазме диода существуют условия для развития неустойчивости Бурсиана-Пирса [15]. Выполненное нами исследование позволит определить предельную величину параметра столкновений, выше которой невозможно возникновение неустойчивости и генератор переменного тока не может функционировать.

\section{Благодарности}

Sourav Pramanik выражает благодарность the Science \& Engineering Research Board (SERB) департамента Науки и Техники Индии за возможность выполнять данные исследования в рамках National Post Doctoral Fellowship (The Fellowship за номером PDF/2017/001132).

\section{Конфликт интересов}

Авторы заявляют, что у них нет конфликта интересов.

\section{Список литературы}

[1] Child C. D. // Phys. Rev. Ser. 1911. Vol. 32. P. 492-511.

[2] Langmuir I. // Phys. Rev. 1913. Vol. 2. P. 450-486.

[3] Бурсиан В.Р.,Павлов В.И. // Жур. Рус. физ.-хим. общ-ва. 1923. Т. 55. Вып. 1-3. С. 71-80.
[4] Pierce J.R. // J. Appl. Phys. 1944. Vol. 15. P. 721-726.

[5] Frey J., Birdsall C.K. // J. Appl. Phys. 1965. Vol. 36. P. 2962-2964.

[6] Coutsias E.A., Sullivan D.J. // Phys. Rev. A 1983. Vol. 27. P. $1535-1543$.

[7] Кузнещов В.И., Эндер А.Я. // ЖТФ. 1977. Т. 47. В. 11. C. 2237-2246.

[8] Mahaffey B.A., Sprangle P., Golden J., Kapetanakos C.A. // Phys. Rev. Lett. 1977. Vol. 39. P. 843--846.

[9] Незлин М.В. Динамика пучков в плазме. М.: Энергоиздат, 1982. $263 \mathrm{c}$.

[10] Akimov P.V., Schamel H., Ender A.Ya., Kuznetsov V.I. // J. Appl. Phys. 2003. Vol. 93. P. 1246-1257.

[11] Ender A.Ya., Kuznetsov V.I., Schamel H., Akimov P.V. // Phys. Plasmas. 2004. Vol. 11. P. 3212-3223.

[12] Ender A.Ya., Kuhn S., Kuznetsov V.I. // Phys. Plasmas. 2006. Vol. 13. P. 113506.

[13] Pedersen A., Manolescu A., Valfells A. // Phys. Rev. Lett. 2010. Vol. 104. P. 175002.

[14] Pramanik S., Ender A.Ya., Kuznetsov V.I. Chakrabarti N. // Phys. Plasmas 2015. Vol. 22. P. 042110.

[15] Бабанин В.И., Колышкин И.Н., Кузнецов В.И., Ситнов В.И., Эндер А.Я. Тр. 2-й отраслевой конф. „Ядерная энергетика в космосе“. Физика термоэмиссионных преобразователей энергии. 1991. Сухуми, Грузия (ФТИ им. Векуа) С. 9-11.

[16] Ottinger P.F., Goodrich P.J., Hinshelwood D.D. et al. // Proc. IEEE. 1992. Vol. 80. N 6. P. 1010-1018.

[17] Garstein Yu.N., Ramesh P.S. // J. Appl. Phys. 1998. V. 83. P. 2958-2964.

[18] Дубинов А.Е., Селемир В.Д. // Радиотехника и электроника. 2002. Т. 47. С. 645-672.

[19] Кузнецов В.И., Соловьев А.В., Эндер А.Я. // ЖТФ. 1994. T. 64. B. 12. C. 9-24.

[20] Ender A.Ya., Kolinsky H., Kuznetsov V.I., Schamel H. // Phys. Rep. 2000. Vol. 328, N 1. P. 1-72.

[21] Akimov P.V., Schamel H., Kolinsky H., Ender A.Ya., Kuznetsov V.I. // Phys. Plasmas. 2001, Vol. 8. P. 3788-3799.

[22] Кузнецов В.И., Эндер А.Я. // Физика Плазмы. 2010. Т. 36. N 2. C. $248-257$.

[23] Кузнецов В.И., Эндер А.Я. // ЖТФ. 2013. Т. 83. В. 12. C. $1-10$.

[24] Schamel H., Bujarbarua S. // Phys. Fluids B. 1993. Vol. 5. P. 2278-22.

[25] Schamel H., Maslov V. // Phys. Rev. Lett. 1993. Vol. 70. P. 1105-1107.

[26] Kolinsky H., Schamel H. // Phys. Plasmas. 1994. Vol. 1. P. 2359-2370.

[27] Kolinsky H., Schamel H. // J. Plasma Phys. 1997. Vol. 57. P. 403-423.

[28] Pramanik S.,Kuznetsov V. I., Chakrabarti N. // Phys. Plasmas. 2015, Vol. 22. P. 082103.

[29] Pramanik S.,Kuznetsov V. I., Chakrabarti N. // Phys. Plasmas. 2018. Vol. 25. P. 083512.

[30] Akimiv P.V., Schamel H. // J. Appl. Phys. 2002. Vol. 92. P. $1690-1698$.

[31] Kuznetsov V. I., Ender A.Ya., Babanin V.I. // J. Appl. Phys. 2018. Vol. 124. P. 044502. 